\title{
PREVALENCIA DE Fasciola hepatica EN BOVINOS (Bos taurus) DEL DISTRITO DE ILABAYA - TACNA
}

\author{
PREVALENCE OF Fasciola hepatica IN CATTLE (Bos taurus) IN THE DISTRICT \\ OF ILABAYA - TACNA
}

\author{
'Teodora Julia Condori Silvestre; ${ }^{2}$ Luis Adolfo Ramos Mamani; ${ }^{3}$ Elizabeth Soledad Chucuya Mamani; \\ ${ }^{4}$ César Orlando Alvarado Calderón
}

\begin{abstract}
RESUMEN
El presente trabajo de investigación se realizó durante el periodo de Abril a Diciembre 2014. Tiene como objetivo determinar la prevalencia de Fasciola hepatica en muestras fecales de bovinos, según clase, sexo y sectores del distrito de llabaya - Tacna. Las muestras fueron procesadas mediante el Método de Sedimentación - Dennis modificado. Se procesó un total de 121 muestras fecales de bovinos, de los cuales, 17 resultaron positivos con una prevalencia de $14,05 \%$. Según clase las vaquillas, terneros y terneras presentaron mayor prevalencia $38,46 \% ; 20,00 \%$ y $18,75 \%$ respectivamente, seguido de las vacas con $10,42 \%$; toretes y vaquillonas con resultados de $8,33 \%$. No hubo presencia del parásito en toros. La prevalencia en hembras fue mayor con el 15,73\% en relación a los machos que sólo fue de 9,38 $\%$. Según sectores los resultados más altos de positividad fueron de 33,33\% en Chululuni y Toco respectivamente, seguido de Cambaya $30,00 \%$, Oconchay $20,75 \%$, Chulibaya 9,09\%, no reportándose casos positivos en llabaya, Ticapampa, Poquera y Mirave. Los resultados nos muestran la existencia de Fascio la hepatica en la población bovina del distrito de llabaya y su presencia podría constituir un serio problema para la salud pública ya que se trata de una zoonosis producida tras la ingestión de metacercarias enquistadas en los pastos que crecen en riachuelos y agua estancada, por lo que dichos lugares son fuente de infección para el hombrey los animales.
\end{abstract}

Palabras clave: Prevalencia, distoma, zoonosis.

\section{ABSTRACT}

This research was done from April to December 2014. Aims to determine the prevalence of Fasciola hepatica in cattle fecal samples, according to: class, sex and places in llabaya district - Tacna. The samples were processed by the Dennis - modified sedimentation method. A total of $121 \mathrm{fecal}$ samples of cattle were processed, from these 17 were positive with a prevalence of $14,05 \%$. According to the class of heifers and calves, they had a higher prevalence $38,46 \% ; 20,00 \%$ and $18,75 \%$ respectively, followed by cows $10,42 \%$; young beef bulls and heifers with results of $8,33 \%$. The presence of the parasite in bulls wasn't found. In females the prevalence was higher $15,73 \%$ compared to $9,38 \%$ males. According to the places, the highest positive results were $33,33 \%$ in Chululuni and Toco respectively, followed by Cambaya $30,00 \%$, Oconchay $20,75 \%$, Chulibaya $9,09 \%$, there weren't positive cases reported in llabaya, Ticapampa, Poquera and Mirave. The results show the existence of Fasciola hepatica in cattle population in the district llabaya and their presence could cause a serious problem for public health, it is a zoonosis produced after the ingestion of metacercaria encysted in the grasses that grow in streams and stagnant water, so these places are a source of infection for humans and animals.

Keywords: Prevalence, fluke, zoonosis.

\section{INTRODUCCIÓN}

La Distomatosis hepática constituye una de la principales enfermedades parasitarias que limitan el desarrollo de la industria pecuaria en el país, ya que los efectos patológicos del distoma se traducen en una disminución notable de la producción y productividad animal, a lo que se suma la pérdida de valiosas fuentes proteicas por el decomiso de hígados parasitados, en el Perú se ha estimado en alrededor de 11 millones de dólares las pérdidas que ocasiona anualmente a la ganadería (Leguía, 1988).

Se ha estimado que en el mundo hay más de 550 millones de estos animales expuestos a sufrir de Distomatosis. Esta afección es causa de decomisos de hígados en frigorifi$\cos$ y de bajas en el potencial productivo de los animales afectados.

Las manifestaciones clínicas dependen de la cantidad y frecuencia de ingestión de metacercarias, pero una constante es el retraso en el crecimiento de los animales, baja producción de carne y leche, mala conversión alimenticia,

Magister en Salud Pública. Médico Veterinario y Zootecnista. Facultad de Ciencias Agropecuarias de la Universidad Nacional Jorge Basadre Grohmann. Tacna-Perú.

${ }^{2}$ Magister en Gestión Ambiental y Desarrollo Sostenible. Médico Veterinario y Zootecnista. Facultad Ciencias Agropecuarias de la Universidad Nacional Jorge Basadre Grohmann. Tacna-Perú.

${ }^{3}$ Médico Veterinario y Zootecnista. Facultad Ciencias Agropecuarias de la Universidad Nacional Jorge Basadre Grohmann. Tacna-Perú.

"Médico Veterinario y Zootecnista. Distrito de llabaya de la provincia Jorge Basadre Grohmann. Tacna-Perú. 
Condori, T. Prevalencia de Fastaola bepatica en bovinos (Bos taurus) del distrito de Ilabaya - Tacna.

pérdida de peso, pérdidas económicas por decomiso de hígados a nivel de matadero y gastos de medicamento para el control, tanto del caracol como del parásito, así como problemas de salud pública en humanos (Rojas, 2004).

Algunos estudios han demostrado diferencias en la resistencia o sensibilidad a esta parasitosis dependiendo de la especie animal. Es así como se ha descrito que el cerdo, el jabalí, el perro y el gato, montan una rápida respuesta contra el parásito evitando su desarrollo. Otro es el caso de los bovinos, los equinos y el hombre que reaccionan en forma tardia, permitiendo su proliferación. Finalmente los ovinos, los caprinos y los lagomorfos son los más receptivos al parásito.

Los ovinos son más susceptibles que los bovinos, y los jóvenes mucho más que los adultos al sufrir esta afección.

El presente estudio es de suma importancia, considerándose que la distomatosis es una enfermedad zoonótica e importante desde el punto de vista de salud pública, debido a las diferentes causas que favorecen su presentación. Estos resultados, van aportar conocimientos a los ganaderos de la zona y otros investigadores sobre la presencia de Distomatosis como enfermedad parasitaria zoonotica y prevenir el riesgo de contagio entre los animales y el hombre y tomar así decisiones para su control y erradicación.

El objetivo de la presente investigación fue determinar la prevalencia general de Fasciola bepatica en bovinos del distrito de Ilabaya-Tacna, teniendo en cuenta la clase, sexo y sectores.

\section{MATERIAL Y MÉTODOS}

\section{Material}

El estudio se realizó en 9 sectores del distrito de Ilabaya, provincia Jorge Basadre, Tacna, entre abril y diciembre del 2014. La zona de estudio se encuentra en los $17^{\circ} 25^{\prime} 23^{\prime \prime}$ de latitud Sur y $70^{\circ} 31^{\prime} 30^{\prime \prime}$ 'de longitud oeste, tiene una extensión superficial de $1111 \mathrm{~km}^{2} \mathrm{y}$ una temperatura promedio por estación de $22,1^{\circ} \mathrm{C}$.

Para la determinación del tamaño de la muestra se usó la ecuación de Cochran; obteniendo una muestra de 121 bovinos. Las muestras fecales de los bovinos fucron recolectadas al azar, y se transportaron con geles refrigerantes para su conservación, hasta el laboratorio de parasitología de la Escuela Académico Profesional de Medicina Veterinaria y Zootecnia de la Universidad Nacional Jorge Basadre Grohmann de Tacna, para su posterior análisis.

\section{Método}

En la evaluación coproparasitológica se utilizó la técnica de sedimentación - Dennis modificado. La muestra fue considerada positiva al observar la presencia del huevo típico de Fasciola bepatica. Los resultados fueron expresados en porcentajes, por lo que se utilizó la fórmula de prevalencia y la prucba de chi cuadrado.

\section{RESULTADOS}

En la tabla 1, se observa que de 121 muestras coprológicas de bovinos examinados, 17 de los cuales resultaron positivas a Fasciola bepatica, con una prevalencia de 14,05\% y 104 muestras fecales resultaron negativos representando $85,95 \%$.
Tabla 1. Prevalencia general de Fasciola bepatica en bovinos (Bostaurus) del distrito de Ilabaya - Tacna.

\begin{tabular}{cccccc} 
& \multicolumn{2}{c}{$\mathbf{N}^{\circ}$} & \multicolumn{2}{c}{ Positivos } & \multicolumn{2}{c}{ Negativos } \\
Especie & Muestra & $\mathrm{N}^{\circ}$ & $\%$ & $\mathrm{~N}^{\circ}$ & $\%$ \\
\hline Bovinos & 121 & 17 & 14,05 & 104 & 85,95 \\
\hline
\end{tabular}

En la tabla 2 se observa que la prevalencia de Fasciola bepatica según clase. Las muestras fecales procedentes de toros resultó negativo a Fasciola bepatica, mientras que en toretes, de un total de 12 muestras fecales examinadas, 01 resultó positivo con una prevalencia de $8,33 \%$; en terneros, de un total de 10 muestras fecales examinadas, 02 resultaron positivos con una prevalencia de $20,00 \%$; en vacas de un total de 48 muestras fecales examinados, 05 resultaron positivos con una prevalencia de $10,42 \%$; de un total de 12 muestras fecales procedentes de vaquillonas, 01 resulto positivo con una prevalencia $8,33 \%$; de 13 muestras fecales procedentes de vaquillas, 05 resultaron positivos con una prevalencia de $38,46 \%$ y de 16 muestra fecales de terneras, 03 resultaron positivas con una prevalencia de $18,75 \%$.

Tabla 2. Prevalencia de Fasciola bepatica por análisis fecal en bovinos (Bos taurus) del distrito de Ilabaya según clase.

\begin{tabular}{lccccc}
\hline & $N^{\circ}$ & \multicolumn{2}{c}{ Positivos } & \multicolumn{2}{c}{ Negativos } \\
Clase & Muestra & No & $\%$ & No & $\%$ \\
\hline Toros & 10 & 0 & 0,00 & 10 & 100,00 \\
Toretes & 12 & 1 & 8,33 & 11 & 91,67 \\
Terneros & 10 & 2 & 20,00 & 8 & 80,00 \\
Vacas & 48 & 5 & 10,42 & 43 & 89,58 \\
Vaquillonas & 12 & 1 & 8,33 & 11 & 91,67 \\
Vaquillas & 13 & 5 & 38,46 & 8 & 61,54 \\
Terneras & 16 & 3 & 18,75 & 13 & 81,25 \\
Total & 121 & 17 & 14,05 & 104 & 85,95 \\
\hline
\end{tabular}

En la tabla 3, se observa que la prevalencia de Fasciola bepática según sexo: De un total de 32 muestras fecales de bovinos del sexo macho, 03 resultaron positivos con un prevalencia de $9,38 \%$, mientras que de un total de 89 muestras fecales de bovinos hembras, 14 resultaron positivos con una prevalencia de $15,73 \%$.

Tabla 3. Prevalencia de Fasciola bepatica por análisis fecal según sexo en bovinos (Bos taurus) del distrito de Ilabaya Tacna.

\begin{tabular}{lccccc}
\hline & $\mathbf{N}^{\circ}$ & \multicolumn{2}{c}{ Positivos } & \multicolumn{2}{c}{ Negativos } \\
Sexo & Muestra & $\mathbf{N}^{\circ}$ & $\%$ & \multicolumn{1}{c}{$\mathbf{N}^{\circ}$} & $\%$ \\
\hline Macho & 32 & 3 & 9,38 & 29 & 90,63 \\
Hembra & 89 & 14 & 15,73 & 75 & 84,27 \\
Total & 121 & 17 & 14,05 & 104 & 85,95 \\
\hline
\end{tabular}

En la tabla 4, se observa que la prevalencia de Fasciola bepatica según sectores del distrito de Ilabaya: En el sector de Chululuni y Toco, la prevalencia de Fasciola bepatica fue del $33,33 \%$ respectivamente; en el sector de Cambaya la prevalencia fue de $30,00 \%$, seguido de Oconchay y Chulibaya con una prevalencia de $20,75 \%$ y $9,09 \%$ respectivamente, no reportándose casos de Fasciola bepatica en el sector de Ilabaya, Ticapampa, Poquera y Mirave. 
Tabla 4. Prevalencia de Fasciola bepatica en bovinos (Bos taurus) según sectores del distrito de Ilabaya - Tacna.

\begin{tabular}{lccccc}
\hline & $N^{\circ}$ & \multicolumn{2}{c}{ Positivos } & \multicolumn{2}{c}{ Negativos } \\
Sector & Muestra & $\mathbf{N}^{\circ}$ & $\%$ & $\mathbf{N}^{\circ}$ & $\%$ \\
\hline Cambaya & 10 & 3 & 30,00 & 7 & 70,00 \\
Chululuni & 3 & 1 & 33,33 & 2 & 66,67 \\
Ilabaya & 13 & 0 & 0,00 & 13 & 100,00 \\
Toco & 3 & 1 & 33,33 & 2 & 66,67 \\
Ticapampa & 5 & 0 & 0,00 & 5 & 100,00 \\
Chulibaya & 11 & 1 & 9,09 & 10 & 90,91 \\
Poqucra & 7 & 0 & 0,00 & 7 & 100,00 \\
Oconchay & 53 & 11 & 20,75 & 42 & 79,25 \\
Mirave & 16 & 0 & 0,00 & 16 & 100,00 \\
Total & 121 & 17 & 14,05 & 104 & 85,95 \\
\hline
\end{tabular}

\section{DISCUSIÓN DE RESULTADOS}

En el análisis de los resultados del presente trabajo de investigación, tenemos que en el distrito de Ilabaya la prevalencia general de Fasciola bepatica fue de 14,05\%.

Sin embargo, Cuenca (2013) obtuvo una prevalencia de $1,16 \%$; Góngora y Santa Cruz (2006) 3,49\%; Torres (2010) 0,3\% y Jiménez (2003) 10,25\%. Al comparar nuestros resultados con los trabajos reportados, se puede observar que la prevalencia es menor en relación a nuestro trabajo, esto debido probablemente al tamaño de muestra, época del año en que se realizó la investigación, y a las condiciones ambientales de las diferentes zonas de estudio.

Narváez (2011) reporta una prevalencia de 24,9\%; Laura (2004) 17,72\%; Sánchez (2009) 16,67\% para Fasciola bepatica; Ramos (2009) 73,31\% y Ticona et al. (2010) 35,9\%. En estos trabajos de investigación se muestran resultados superiores a los obtenidos en nuestro trabajo de investigación, csto debido probablemente al deficiente manejo sanitario que se refleja en el parasitismo de sus animales.

En el presente trabajo de investigación, la prevalencia fue mayor en animales jóvenes: terneros $20,00 \%$; vaquillas $38,46 \%$ y en terneras $18,75 \%$, que son más susceptibles al parasitismo en comparación con los adultos, que son más resistentes.

Mientras Jiménez (2003) reportó una prevalencia para vacas $12,43 \%$, vaquillas $5,55 \%$; terneras $6 \%$; toros $12,50 \%$; toretes $17,39 \%$; terneros $4,87 \%$. Sin embargo, Laura (2004) reportó que la prevalencia para toros fue de $26,47 \%$, torete $27,59 \%$; ternero $6,90 \%$; vaca $17,24 \%$; vaquilla $13.04 \%$, ternera $19,05 \%$. Sánchez (2009) reportó según clase animal, que la prevalencia de Fasciola para terneras fue de $3,57 \%$; vaquillas $11,11 \%$; vaquillona $31,58 \%$; vaca $19,85 \%$; ternero $10,53 \%$; torete $0,0 \%$; y toro $11,11 \%$. Estos trabajos reportaron resultados que difieren de los nuestros, se puede observar que existe una mayor prevalencia en animales adultos, esto probablemente al tipo de alimentación y a la exposición en zonas muy contaminadas por Fasciola bepatica, produciendo en ellos huéspedes perfectos para su desarrollo y diseminación.

En nuestro trabajo de investigación la prevalencia de Fasciola bepatica en bovinos según sexo fue mayor en hembras $15,73 \%$, siendo menor en machos $9,38 \%$.
Al comparar nuestros resultados, se observó similitud con investigaciones reportados por: Góngora y Santa Cruz (2006) en bovinos machos con el 2,86\% y en hembras con el 7,43\%. Torres (2010) reporta que solo las hembras presentaron una prevalencia de $0,3 \%$, mientras que los machos no presentan la incidencia de esta enfermedad. Jiménez (2003) menciona que en hembras fue de 10,39 \% y machos $9,72 \%$. Sánchez (2009) indica que la prevalencia fue para hembras $14,96 \%$ y para machos $1,71 \%$. Ramos (2009) obtuvo, según sexo, que los machos presentan una prevalencia de $70,15 \%$ y las hembras de $77,42 \%$ y Ticona et al. (2010) $36,7 \%$ y $34,0 \%$ para hembras y machos respectivamente.

Sin embargo nuestros resultados difieren con los reportados por Laura (2004), en la Provincia de Candarave, quien obtuvo una mayor prevalencia para bovinos machos $(32,20 \%)$ y menor en hembras $(26,64 \%)$.

Los resultados para la prevalencia de Fasciola bepatica en los bovinos del distrito de Ilabaya según sectores fueron de $33.33 \%$ para Chululuni y Toco. En Cambaya la prevalencia fue de $30,00 \%$, seguido de Oconchay y Chulibaya con una prevalencia de $20,75 \%$ y $9,09 \%$ respectivamente. No reportándose casos de Fasciola bepatica en el sector de Ilabaya, Ticapampa, Poquera y Mirave.

\section{CONCLUSIONES}

Las conclusiones a las que se llega al finalizar el presente trabajo de investigación, en concordancia con los objetivos son las siguientes: I. a prevalencia general de Fasciola bepatica en bovinos del distrito de Ilabaya es de 14,05\%. Según clase, en toros fue $0,00 \%$; toretes $8,33 \%$; terneros $20,00 \%$; vacas $10,42 \%$; vaquillonas $8,33 \%$; vaquillas 38,46 $\%$ y terneras $18,75 \%$. La mayor prevalencia fue en hembras $15,73 \%$ en relación a los machos $9,38 \%$.

Según sectores fue: Cambaya 30,00\%; Chululuni $33,33 \%$; Toco 33,33 \%; Chulibaya 9,09 \%; Oconchay $20,75 \%$. No reportándose presencia del parásito en Ilabaya, Ticapampa, Poquera y Mirave.

\section{REFERENCIAS BIBLIOGRÁFICAS}

Cali, N. (2012). Incidencia de Fasciola bepatica en las empresas de rastro de la provincia de Chimborazo. Tesis Ingeniero zootecnista. Escuela superior politécnica de Chimborazo. Riobamba, Ecuador.

Cuenca, E. (2013). Prevalencia de Fasciola bepatica en bovinos sacrificados en el cantón machala. Tesis Médico Veterinario. Ecuador. Universidad Técnica de Machala, Ecuador.

Cordero del Campillo, M. y Rojo, F. (1999). Parasitologia Veterinaria, Edit Mc GRAW-HILL. Interamericana. España. Pág. 260-271

Góngora, R.C. y Santa Cruz, G.S. (2006). Prevalencia de Fasciola bepática en bovinos faenados en el matadero municipal de la ciudad de la Paz, Octubre 2005 a marzo 2006. Tesis Médico Veterinario y Zootecnista. Santa Cruz de la sierraBolivia. Facultad de Ciencias Veterinarias, UAGRM.

Jiménez, S.D. (2003). Prevalencia de distomatosis bepática en el Valle de Sama-Tacna. Tesis Médico Veterinario y zootecnista. UNJBG. 60 pág. 


\section{Ciencia \& Desarrollo}

Condori, T. Prevalencia de Fasciola bepatica en bovinos (Bos taurus) del distrito de Ilabaya - Tacna.

Kassai, T. (2002). Helmintologia veterinaria. Edit. Acribia S.A. España. Pág. 4-12.

Laura, M, L. (2003). Determinación de la prevalencia de distomatosis hepaática en vacunos de la provincia de Candarave-Tacna. Tesis Médico Veterinario y Zootecnista. UNJBG.

Leguia, G. (1988). Distomatosis hepática en el Perú, epidemiología y control. Rev. Hoechst. Lima-Perú. 32 pag.

Narváez, A. (2011). Prevalencia y factores asociados a la Fasciola bepatica y otras parasitosis intestinales en la comunidad de Tarqui-2011. Ecuador. Tesis Master en epidemiologia. 41 pág.

Quiróz, H. (2009). Parasitologia y enfermedades parasitarias de animales domésticos. Edit. LIMUSA. México. Pag. 876.

Rojas, M. (2004). Nosoparasitosis de los rumiantes domésticos peruanos. 2da Edición. Lima-Perú. Pág. 37-42.

Ramos, F.J. (2009). Evaluación económicapor el decomiso de bigados de bovinos con fascioliasis en el camal municipal de Moquegua. Tesis Médico Veterinario y zootecnista. UNJBG. Tacna, Perú.

Sánchez, L. J. (2009). Evaluación parasitaria del ganado vacuno (Bos Taurus) en el distrito de Ite-Tacna. Tesis Médico Veterinario y zootecnista. UNJBG. 90 pag.

Soulsby, E. (1988). Parasitologia y enfermedadesparasitarias. Edit. Interamericana. México. Pag. 823 37-49.

Ticona, D., Chávez, A., Casas, G., Chavera, A. y Li, O. (2010) Prevalencia de Fasciola bepatica en bovinos y ovinos de Vilcashuamán, Ayacucho. Rev. investig. vet. vol 21, núm. 2.

Torres, P.J. (2010). Incidencia de fascioliasis bepática en bovinos faenados en el camal municipal de la ciudad de Bababoyo - Los Rios - Ecuador. Tesis Médico Veterinario y Zootecnista. Universidad Técnica de Babahoyo. 72 pág.

\section{Correspondencia:}

Teodora Julia Condori Silvestre: juliacondori@yahoo.com

Fecha de Recepción: 15/04/2014

Luis Adolfo Ramos Mamani: larm_unjbg@hotmail.com

Fecha de Aceptación: 01/06/2015

Elizabeth Soledad Chucuya Mamani: soledad041083@gmail.com

César Orlando Alvarado Calderón: nand_ito@hotmail.com 